\title{
Übersicht
}

\section{„Sportanämie“ - Wieviel Blut kostet ein Marathonlauf?}

\author{
"Sports Anemia" - How Much Blood is Used up During a Marathon Race?
}

\author{
L. Röcker ${ }^{1,2}$, Christiane Röcker ${ }^{3}$
}

Zusammenfassung: Ausdauerleistungen führen akut und chronisch z.u deutlichen hämatologischen Veränderungen. Während es bei akuten Laufleistungen zu ciner transitorischen intravaskulären Hämolyse konınit, findet man bei chronischen Ausdauerleistungen (d.h. bei Trainierten) ernicdrigte Konzentrationen von Hämoglobin und roten Blutzellen. Dieses Phänomen vird häufig als "Sportanämie" bezeichnet. Um den Einfluß der Hämolyse auf das Phänomen ..Sportanämie“" zu erkennen, wurde der Blutverlust nach verschiedenen Marathonläufen anhand von eigenen und publizierten Daten abgeschätzt. Dieser Blutverlust erwies sich mit 4.6-13 ml als sehr gering und kann deshalb nicht für das bei Ausdauersportlern übliche subnormale rote Blutbild verantwortlich sein. Vielmehr ist die Ursache dafür mit dem unverhältnismäßig vergrößerten Plasmavolumen zu erklären, das zu einer Verdünnung des Gesamtblutes führt. Die absolute Zahl der roten Blutzellen ist beim Ausdauertrainierten erhöht, deshalb sollte dieses Phänomen nicht mit „Sportanämie“" bezeichnet werden, da es sich um eine Anpassung an die körperliche Leistung oder nur um ein Epiphänomen handelt. Die Bezeichnung Pseudoanämie ist hier vorzuziehen.

Schlüsselwörter: Anämie; Hämolyse; Ausdauersport/Physiologie.

Summary: Acute and chronic physical exercise induces marked hematological changes. While after acute endurance exercise hemolysis is regularly found, the adaptation to endurance exercise training results in subnormal values of red blood cells, hemoglobin and hematocrit values. The phenomenon is frequently called "sports anemia". The blood loss from exercise induced hemolysis was quantitatively estimated after marathon races (from several published studies and own experiments), to establish whether it is the cause of "sports anemia". The quantitative blood loss was very small $(4.6-13 \mathrm{ml})$ and cannot be made responsible for the subnormal red blood cell count and hemoglobin / hemotocrit values seen in endurance athletes.

\footnotetext{
1 Physiologisches Institut, Freie Universität Berlin

2 Korrespondenzadresse: Prof. Dr. Lothar Röcker, Physiologisches Institut, Freie Universität Berlin, Arminallee 22, D-14195 Berlin. Fax: +49-30-802 4647

3 Medizintechnische Vertriebs-GmbH Berlin

Eingegangen: 19. August 1998/Angenommen: 20. November 1998
}

The causal mechanism of this phenomenon can be attributed to the disproportional larger plasma volume in endurance athletes resulting in a dilution of the increased total number of red blood cells. Therefore this phenomenon should be designated as pseudoanemia rather than "sports anemia". It is an adaptation to endurance exercise or only an epiphenomenon.

Keywords: Anemia; Hemolysis; Physical Endurance/ physiology.

A usdauersport, vor allem Marathonlauf, ist heutzutage ein populärer Sport gevvorden. Ausdauertraining führt zu vielfältigen Anpassungen und Veränderungen der verschiedenen Organsysteme des menschlichen Organismus, insbesondere des Herz-KreislaufSystems und des Muskelsystems.

In dem vorliegenden Beitrag soll das seit langem bekannte Phänomen der sogenannten Sportanämie. insbesondere der Blutverlust beim Marathonlauf durch das Auftreten einer intravaskulären Hämolyse, genauer betrachtet werden.

\section{Hämolyse - Haptoglobin}

Im Jahre 1938 fanden Polonovski und Jayle [1] im menschlichen Plasma einen Eiweißkörper, der die Fähigkeit besitzt, freies Hämoglobin irreversibel. zu binden.

Dieser Eigenschaft hat das Plasmaprotein seinen Namen Haptoglobin zu verdanken. 1955 wies Smithies [2] die Existenz von drei Haptoglobin-Phänotypen nach: Haptoglobin 1-1, 2-1 und 2-2, die sich im wesentlichen in ihren Molekulargewichten unterscheiden. Es ist entwicklungsgeschichtlich interessant, da $\beta$ die polymeren Phänotypen 2-1 und 2-2 nur beim Menschen zu finden sind. Da der Mensch Jahrtausende lang als Jäger und Sammler große Laufstrecken zu bewältigen hatte, vermutet man. daß der Haptoglobinpolymorphismus einen Selektionsvorteil gebracht hat. Während das relativ kleine Hämoglobinmolekül nierengängig ist, wird der Hämoglobin-HaptoglobinKomplex wegen seines größeren Molekulargewichtes, insbesondere bei den polymeren Typen 2-1 und 2-2. nicht über die Nieren ausgeschieden. Folglich ist der Organismus vor Eisenverlusten weitgehend geschützt. Sobald sich im Plasma der Hämoglobin-Haptoglobin- 
Komplex gebildet hat, wird dieser vom retikuloendothelialen System (RES) aufgenommen [3] und somit aus dem intravaskulären Raum entfernt (,,suicide protein"). Aufgrund dieses Mechanismus ist die Haptoglobinkonzentration in allen Situationen, in denen intravaskuläre Hämolysen auftreten, je nach deren Schweregrad erniedrigt oder überhaupt nicht mehr nachweisbar. Damit ist der Schutzmechanismus gegen Eisenverluste vorübergehend eingeschränkt oder unter Umständen gar nicht mehr existent, so daß Hämoglobin über die Nieren ausgeschieden wird. In der Labordiagnostik gilt die Erniedrigung der Haptogiobinkonzentration als ein zuverlässiger Hämolyseindikator (Ausnahme bei den in Europa seltenen genetischen Defekten einer Ahaptoglobinämie). Im Vergleich zur Bestimmung von freiem Hämoglobin und anderen Indikatoren der Hämolyse wird Haptoglobin nicht durch in-vitro Hämolysen beeinflußt.

\section{Hämolyse und Ausdauersport}

Der Erythrozyt hat eine begrenzte Lebensdauer von 120 Tagen, das entspricht einer Zirkulationsstrecke von ca. $200 \mathrm{~km}$. Etwa 0,8\% aller Erythrozyten werden normalerweise in 24 Stunden abgebaut und durch Retikulozyten ersetzt. Das sind pro Tag ca. 200 Milliarden Erythrozyten oder etwa $40 \mathrm{ml}$ Blut [4]. Der physiologische Abbau erfolgt überwiegend extravasal im retikulo-histiozytärem System von Milz, Leber und Knochenmark. Nur 10 - 20\% werden intravaskulär abgebaut; das frei werdende Hämoglobin wird über den Haptoglobinmechanismus eliminiert. Unter bestimmten pathologischen Bedingungen ist die Lebenszeit der Erythrozyten verkürzt, dieses Phänomen wird als intravaskuläre Hämolyse bezeichnet [5].

Akute Ausdauerleistung löst deutliche Veränderungen des.peripheren roten Blutbildes aus [6]. Unmittelbar nach akuten körperlichen Ausdauerleistungen beobachtet man in Abhängigkeit von Intensität, Trainingszustand, Wasserbilanz, etc. meist einen transitorischen Anstieg (10-30\%) des Hämatokritwertes, der Hämoglobin- und Erythrozytenkonzentration $[4,6]$. Bis zu einer Woche nach der Leistung findet man dann sogar erniédrigte Werte. Generell weisen Ausdauersportler im Vergleich zu Untrainierten subnormale Werte des peripheren roten Blutbildes auf.

Seit über 100 Jahren ist bekannt, daß es bei lang dauernden körperlichen Leistungen zu einer intravaskulären Hämolyse kommt, manchmal sogar zu einer Hämoglobinurie [7-15]. Das bei Laufleistungen aus den Erythrozyten freigesetzte Hämoglobin wird im Plasma an Haptoglobin im Verhältnis 1:1 gebunden und konsekutiv im RES abgebaut. Deshalb ist Haptoglobin im Plasma nach einer länger dauernden Lauf-

Nicht standardisierte Abkürzungen: EPO.. Erythropoetin; KGW, Körpergewicht; PV, Plasmavolumen; RES, retikuloendotheliales System: TrR, Transferrinrezeptor. leistung mehr oder weniger erniedrigt oder sogar nicht mehr nachweisbar.

Obwohl die Literatur über Hämolysezeichen und Haptoglobinveränderungen beim Sport sehr umfangreich ist, gibt es u. E. keine quantitative Abschätzung des Haptoglobinverbrauchs und den sich daraus ergebenden Hämoglobin- bzw. Blutverlusten bei einem Marathonlauf oder anderen Ausdauerleistungen.

Dies liegt daran, daß wichtige Größen für die Berechnung des Blutverlustes im allgemeinen nicht bestimmt wurden. Um den Blutverlust quantitativ abschätzen zu können, muß das Plasmavolumen (PV) bekannt sein. Denn aus PV und Proteinkonzentration lassen sich diverse intravaskuläre Mengen (Menge $=\mathrm{PV}$ $x$ Konzentration) von Einzelproteinen, z. B. die Haptoglobinmenge, berechnen [16].

-In Tabelle 1 sind aus mehreren Publikationen die intravaskulären Blutverluste (Erythrozytenverluste) nach Marathonläufen aus den angegebenen Haptoglobinwerten und den gemessenen oder berechneten Plasmavolumina abgeschätzt worden. Bei den zitierten Arbeiten erfolgte die Bestimmung des PV nach dem Indikatorverdünnungsprinzip [14]. Als Indikatorsubstanz diente ${ }^{125} \mathrm{~J}$ - bzw. ${ }^{131} \mathrm{~J}$-markiertes Humanserum-Albumin. In den Literaturangaben, bei denen kein

PV bestimmt oder angegeben wurde $[8,12,17,18]$, erfolgte die Berechnung des PV aus den Regressionsgleichungen von PV und Körpergewvicht bzw. Körpergröße, die von Wennesland 1959 [19] aufgestellt wurden. Die Veränderungen des PV nach der Leistung wurden aus den angegebenen mittleren Hämoglobinund Hämatokritkonzentrationen nach der Formel von Strauss et al. [20] berechnet. Diese Methode ist in der Sportphysiologie'seit Jahren üblich.

Die Hämoglobin-Bindungskapazität [21] des Plasmas (Haptoglobin) wurde bei diesen Untersuchungen nicht überschritten. Deshalb läßt sich aus der Haptoglobinverminderung und des PV die Hämolyse quantitativ abschätzen, d.h., die Menge an Hämoglobin bzw. der Blutverlust berechnen, der bei einem Marathonlauf auftritt.

Wie aus Tabelle 1. ersichtlich, ist der abgeschätzte intravaskuläre Blutverlust nach einem Marathonlauf außerordentlich gering [ 4,6 bis $13,0 \mathrm{ml}]$ und kann deshalb nicht die Ursache für das bei Ausdauersportlern bekannte subnormale rote Blutbild sein (s.u.).

Diese geringe Menge an Erythrozyten (21 Milliarden bis 59 Milliarden) kann schnell durch eine erhöhte Erythropoese nach einem Marathonlauf wieder ausgeglichen werden. Dies geschieht durch das Hormon Erythropoetin (EPO), das der Hauptregulator der Erythrozyten ist. So wurden nach Marathonläufen erhöhte Erythropoetinspiegel im Blut gefunden [22]. Ausgehend von einem mittleren Kontrollwert von 18,3 $\mathrm{mU} / \mathrm{ml}$ waren die EPO-Spiegel 3 Stunden nach dem Lauf um $1,4 \mathrm{mU} / \mathrm{ml}(\mathrm{p}<0,05)$ und 31 Stunden nach dem Lauf um 5;7 mU/ml ( $<<0,001)$ angestiegen.

Die Ursache für die transitorische Hämolyse beim Sport ist noch nicht restlos geklärt. Eine interessante Hypothese lieferte Davidson [7], nach dessen Vorstel- 
lung die Hämolyse in der Mikrozirkulation der Fubsohlen durch mechanische Eintvirkungen des Körpergewichts auf dic Erythrozyten entsteht (hïufig als .foot-strike hemolysis" be\%cichnet). Aber das scheint nicht der cinsige Wirkungsmechanismus der Hämolyse zu scin. Seihy und Eirhner |23| zeigten, daß auch nach anstrengenden Schivimmleistungen cine intravaskulïre Hämolyse auftrat, wobei keine mechanischen Einwirkungen in den Fußsohlen auftreten. Vielmehr könnten auch Turbulenzen in der Blutströmung zu cincr Hämolyse führen 124]. Weiterhin beschrieben Yoshimura et al. [25]. daß3 Erythrozyten durch einen Hämolysefaktor (Ly'solezithin) zerstört werden, der bei anstrengenden Leistungen aus der Milz freigesetzt wird. Eine weitere, bisher noch nicht beschriebene Ursiache könnte folgende sein: Bei körperlichen Leistungen ist die Wegstrecke der Erythrozyten von $200 \mathrm{~km}$ durch die erhöhte Zirkulation schneller erreicht, so daß die Überlebenszeit insgesamt verringert ist, der Zelluntergang jedoch beschleunigt.

Weight und Mitarbeiter [26] fanden, daß3 bei gut trainierten Ausdauerläufern die Überlebenszeit der Erythrozyten kürzer war als bei Untrainierten. Ober ähnliche Ergebnisse berichten auch andere Autoren $[27,28]$.

Möglicherweise wird eine Subpopulation älterer Erythrozyten bevorzugt aus der Zirkulation eliminiert [29]. Aber auch die erhöhte Körpertemperatur, die Azidose. erhöhte Katecholaminiverte beim Ausdauersport könnte zu Membranveränderungen und konsekutiver Hämolyse führen. Weiterhin könnte die Hämolyse auf einer Destruktion von Erythrozyten in der Endstrombahn der sich kontrahierenden Skelettmuskulatur zurückzuführen sein [9].

Während hämolytische Veränderungen nach allen Ausdauerläufen nachiveisbar sind, ist das Auftreten einer Hämoglobinurie die Ausnahme. Sie tritt nämlich erst auf, wenn die Hämoglobinbindungskapazität des Plasmas (speziell von Haptoglobin) überschritten ist.

\section{„Sportanämie“" subnormales rotes Blutbild}

In zahlreichen Untersuchungen wurde bei Ausdauertrainicrten im Vergleich zum Nichtsportler eine erniedrigte Erythrozyten- und Hämoglobinkonzentration nachgewiesen $[4,14,15,30\}$. Dieses Phänomen wird auch mit dem verwirrenden Begriff „Sportanämie“ belegt.

Referenzbcreiche für die Subpopulation der Ausdauersportler wurden nach unserer Kenntnis bisher nicht aufgestellt. Bei Vergleichsuntersuchungen zwischen Ausdauertrainierten und Untrainierten wurde ein um ca. $5 \mathrm{~g} / \mathrm{l}$ verminderter Hämoglobinwert und ein um ca. $2 \%$ verminderter Hämatokritwert beobachtet [31, 32].

Wie häufig an biologischen Systemen zu beobachten ist, sind auch hier Konzentrationen und absolute Mengen nicht gleichsinnig verändert.

Die Gesamterythrozytenmenge und die Hämoglobinmenge sowie das Plasmavolumen sind bei Ausdauertrainierten im Vergleich zu Nichtsportlern erhöht. Demzufolge ist auch das Gesamtblutvolumen bei Ausdauersportlern größer. Beide Blutvolumenanteile werden jedoch von verschiedenen Mechanismen kontrolliert.

Da das Plasmavolumen bei Ausdauertrainierten im Vergleich zum Erythrozytenvolumen überproportional vergrößert ist (PV: $+485 \mathrm{ml}$ versus EV: $+334 \mathrm{ml}$ ) [14] resultiert eine verminderte Konzentration (subnormal) der Erythrozyten und des Hämoglobins.

Die unverhältnismäßige Erhöhung des Plasmavolumens bei Ausdauersportiern ist teleologisch betrachtet als positiv einzuschätzen, da der erniedrigte Hämatokritwert die Viskosität des Gesamtblutes vermindert $[32,33,34,35]$ und damit die Herzarbeit. Außerdem spielt eine Vergrößerung des Plasmavolumens für die Thermoregulation und die Erhaltung ihrer Homöostase eine wesentliche Rolle. Über zirkadiane Schwankun-

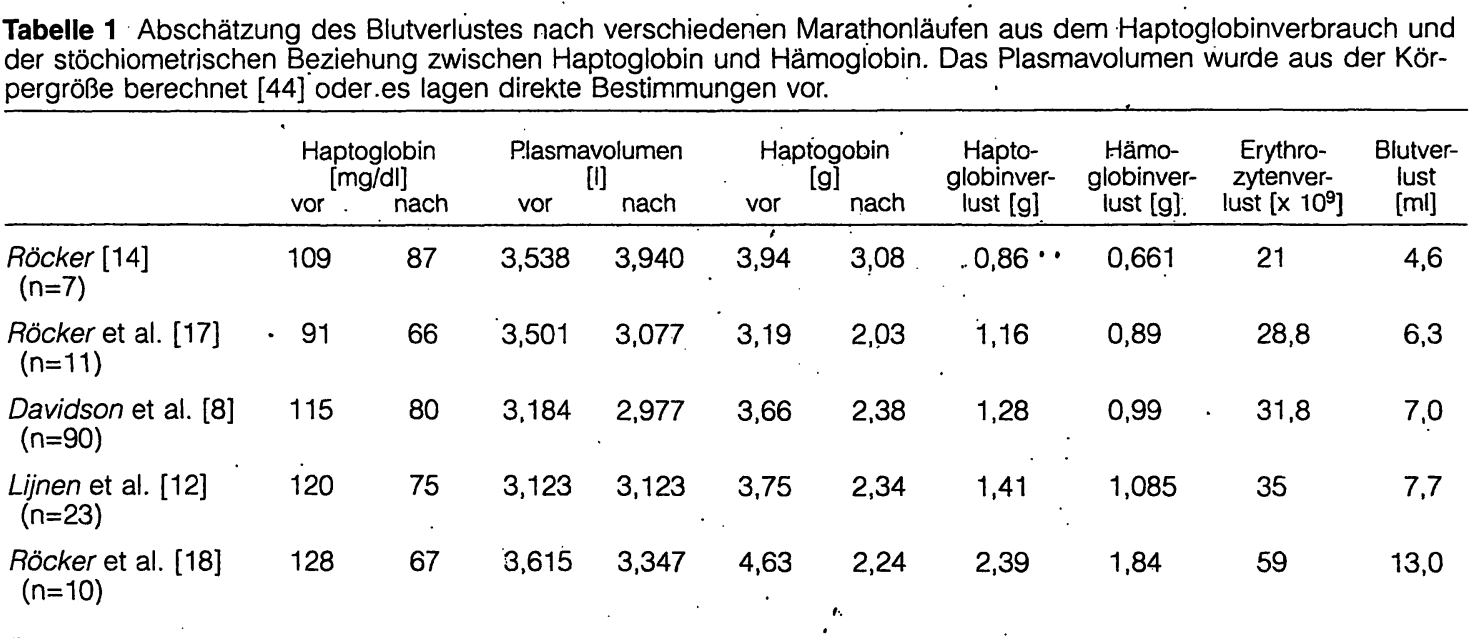


gen des Plasmavolumens gibt es nur sehr wenige Untersuchungen [36]. Dabei zeigte sich eine geringe interindividuell konstante Rhythmik mit einem Maximum um $0.00 \mathrm{Uhr}(+5 \%)$ und einem Abfall bis zum Minimum um 8.00 Uhr $(-4 \dot{\%})$. Die Veränderung der Plasmaproteine entspricht in der Amplitude den PVVeränderungen, sie ist jedoch in ihrer Ausrichtung der PV-Veränderung entgegengesetzt.

Die subnormalen Konzentrationswerte bezüglich des roten Blutbildes bei Ausdauersportlern dürfen nicht in Sinne einer Anämie fehlinterpretiert werden, wie dies gelegentlich diskutiert wird [25, 37], da absolut gesehen, die Sauerstofftransportkapazität vergröBert ist und es sich im allgemeinen um einen Verdünnungseffekt des überproportional vergrößerten Plasmavolumens handelt.

Eine bessere Bezeichnung für das subnormale rote Blutbild beim Ausdauersportler ist mit dem Begriff „Pseudoanämie“" getroffen [38]. Vor allem kommt diesem Phänomen kein Krankheitswert zu [9]. Bei der Pseudoanämie handelt es sich wahrscheinlich um eine Anpassung an Ausdauersport oder auch nur um ein Epiphänomen.

\section{Anämie-Diagnostik beim Ausdauerläufer}

Unabhängig von dem subnormalen roten Blutbild kann sich auch bei Ausdauersportlern eine Anämie entwickeln.

Die Gesamtmenge der Erythrozyten im Blut wird unter physiologischen Bedingungen von dem Hormon Erythropoetin reguliert. Eine Verminderung der $\mathrm{Ge}$ samtmenge der Erythrozyten bedeutet Anämie [39].

Die Feststellung einer Anämie, vor allem im Grenzbereich, ist zunächst durch einen Laborbefund gekennzeichnet, der einer individuellen Wertung bedarf. Anämien verden in den meisten Fällen durch eine erniedrigte Hämoglobinkonzentration erfaßt, daneben wird der Hämatokritwert und die Erythrozytenkonzentration zur Diagnostik herangezogen. Besonders beim Ausdauersportler hat die Interpretation des peripheren roten Blutbildes die Änderungen des absoluten Erythrozytenvolumens und des PV zu berücksichtigen. Bei Ausdauersportlern kommt es zu einem Verdünnungseffekt des peripheren roten Blutbildes, der nichts mit einer Anämie zu tun hat $[15,16,38]$. Die üblichen Referenzbereiche für das rote Blutbild können deshalb nicht zur Beurteilung herangezogen werden. Eine bessere Beurteilung liefert die direkte Messung des absoluten Erythrozytenvolumens, welches beim Erwachsenen normalerweise $27-33 \mathrm{ml} / \mathrm{kg} \mathrm{KGW}$ beträgt [19]. $\mathrm{Da}$ die Blutvolumenbestimmungen nur in Spezialinstituten durchgeführt wird und daher unpraktikabel ist, sollten die von Eichner [40] angegebenen Werte als Richtgrößen für eine klinisch relevante Anämie beim Sportler verwendet werden: Hämoglobin bei Frauen $<110 \mathrm{~g} / \mathrm{l}$, bei Männern < $130 \mathrm{~g} / \mathrm{l}$.

Eine ähnliche Situation wie beim Ausdauersportler beobachtet man in der Schwangerschaft: Nach Ablauf des ersten Trimenon nimmt das Erythrozytenvolumen - bei optimaler Eisenzufuhr - um durchschnittlich $25 \%$ zu, das PV jedoch um $40 \%$ [41].

Die häufigste Ursache einer Anämie beim Ausdauersportler, insbesondere bei Ausdauersportlerinnen, ist der Eisenmangel. Dies liegt u.a. daran, daß Leistungssportler einen erhöhten Eisenbedarf haben. Ein Eisenmangel hat schon vor dem Auftreten einer Anämie Symptome wie Leistungsminderung zur Folge [31].

Auch hier gestaltet sich die Diagnostik schwierig, da die Ferritinbestimmung als klassischer Marker zur Feststellung eines Körpereisenmangels versagen kann. Als ,akute Phase“ Protein ist das Ferritin noch 3 Tage nach einem Marathonlauf falsch hoch zu finden. Andererseits findet man bei Ausdauertrainierten im Vergleich zur Normalbevölkerung häufig niedrigere Ferritinwerte $[42,43]$. Nach einem intensiven 4wöchigen Trainingsprogramm sanken die Ferritinwerte um 50\% bei beiden Geschlechtern [44]. Bei der Beurteilung des Eisenstoffwechsels ist zu beachten, in welcher Trainingsphase die Bestimmung der Laborbefunde erfolgt. Im Ziveifelsfalle sollte man die Bestimmungen nach einer 10tägigen Regenerationspause durchführen oder wiederholen, da nach dieser Zeit die Ferritinwerte wieder ihren Ausgangswert erreichten [42]. In Zukunft könnte die Bestimmung der löslichen Transferrinrezeptoren (TrR) im Serum zur Diagnostik eines Eisenmangels beim Sportler ein zusätzlicher oder geeigneterer Indikator sein. Der $\operatorname{TrR}$ ist ein transmembranes Glykoprotein, das die Aufnahme von Transferrin-Eisen in die Körperzellen kontrolliert. Bei Eisenmangel zeigt der lösliche TrR und Serumferritin ein unterschiedliches Verhalten. Ferritin erfaßt den Gehalt an Speichereisen, dagegen zeigt der TrR den Grad des Eisenbedarfs im Gewebe direkt an. Im Vergleich zum Serumferritin stellte sich heraus, daß der TrR unter bestimmten Bedingungen (falsch hohes oder falsch niedriges Ferritin) der zuverlässigere Parameter bei der Diagnose einer Eisenmangelanämie ist [47].

Die TrR-Konzentration soll unabhängig von einer akuten Phase-Reaktion sein, es bestehen keine Geschlechtsunterschiede und in der Schwangerschaft bleiben die Konzentrationen im Gegensatz zu Ferritin unverändert $\left[45^{\circ}, 46\right]$. Es liegen noch zu wenig Erfahrungen vor, um ein abschließendes Urteil über den Wert von TrR-Bestimmungen beim Ausdauersportler abzugeben.

\section{Literatur}

1. Polonovski $M$, Jayle $M$. Existence dans le plasma sanguin d'une substance activant l'action peroxydasique de l'hämoglobine. Compt Rend Soc Biol Paris 1938;129:457-60.

2. Smithies 0. Zone electrophoresis in starch gels: Group variations in the serum proteins of normal human adults. Biochem $J$ 1955;61:629-41.

3. Putnam FW. Alpha, Beta, Gamma, Omega - The roster of the plasma proteins. In: Putman FW, editor. The Plasma Proteins, Structure, Function and Genetic Control. 2. ed. Vol 1. New York (USA): Academic Press, 1975: 57-131.

4. Szygula $Z$. Erythrocytic system under the influerence of physical exercise and training. Sports Medicine 1990;10: 181-97. 
5. lïscher IT. Die Diagnose der hämolytischen Anämic. Internis Welt 1):0:3:31-9.

6. Röcker 1 .. Der Einfluß körperlicher Leistungen auf Laborbefuncle. Kassenarzl 1983:23:35-44.

7. Davidson. RJ. lixcrtional hacmoglohinuria: a report on three cases with studies on the haemolytic mechanism. J. Clin Path $1964: 17: 536-40$.

8. Davidson RJ. Rohertson JD. Galea G. Maughan RJ. Haematological changes associated with marathon runninng. Int J Sports Med 1987:8:9-25.

9. Ernst 1:. Sturmvoll M. Magyarcosi I. ..Sportanämic" - Wohin verschwinden dic Erythrozyten? Disch Zeitschr Sportmedizin 1988:39:476-80.

10. Fleischer R. Über eine neue Form von Hacmuglobinuric beim Menschen. Berlin Klin Wschr 1881:18:691-4.

11. Gilligan DR. Altschule MD, Katersky EM. Physiological intravascular hemolysis of exercise. Hemoglobinemia and hemoglobinuria following cross-country runs. J Clin Invest 1943:22:859-69. 12. Lijnen P. Hespel P. Fagard R, Lysens R. Vanden Eynde E. Goris M. Goossens WV. Lissens W, Amery A. Indicators of cell breakdown in plasma of men during and after a marathon race. Int J Sports Mled 1988:9:108-13.

13. Miller BJ, Pate RR. Burgess W. Foot impact force and intravascular hemolysis during distance running. Int J Sports Med 1988;9:56-60.

14. Röcker L. Das Verhalten von Plasmavolumen und Plasmaproteinen nach körperlichen Leistungen, körperlichem Training und Hitzeeinwirkung. Habilitationsschrift. Berlin 1978.

15. Röcker L. Laniado M, Kirsch K. The effect of physical exercise on plasma volume and red blood cell mass. In: Dunn CDR, editor. Current concepts in erythropoiesis. Chichester, New York, Brisbane. Toronto, Singapore (England): John Wiley \& Sons Ltd. 1983:245-77.

16. Röcker L. Kirsch KA. Stoboy H. Plasma volume, albumin and globulin concentrations and their intravascular masses. A comparative study in endurance athletes and sedentary subjects. Eur J Appl Physiol 1976:36:57-64.

17. Röcker L. Drygas WK. Heyduck B. Blood platelet activation and increase in thrombin activity following a marathon race. Eur $J$ Appl Physiol. 1986;55:374-80.

18. Röcker L. Tänzer M. Drygas WK, Lill H, Heyduck B, Altenkirch HU. Effect of prolonged physical exercise on the fibrinolytic system. Eur J Appl Physiol 1990;60: 478-81.

19. Wennesland R, Brown E, Hopper J Jr, Hodges JL Jr, Guttentag OE. Scott KG. Tucker, IN, Brandley B. Red cell, plasma and blood volume in healthy men measured by radiochromium $(\mathrm{Cr} 51)$ cell tagging and hematocrit: Influence of age, somatotype and habits of physical activity on the variance after regression of volumes to height and weight combined. J Clin Invest 1959;38: 1065-77.

20. Strauss MB, Davis RK, Rosenbaum JD, Rossmeisl EC. "Water diuresis" produced during recumbency by the intravenous infusion of isotonic saline solution. J Clin Invest 1951;30:862-8.

21. Laurell CB, Nyman M. Studies on the serum haptoglobin level in hemoglobinernia and the influence on renal excretion of hemoglobin. Blood 1957;12:493-506.

22. Schwandt HA, Heyduck B, Gunga HC, Röcker L. Influence of prolonged physical exercise on the erythropoietin concentration in blood. Eur J Appl Physiol 1991:63:463-6.

23. Selby GB. Eichner ER. Endurance swimming, intravascular hemolysis, anemia and iron depletion. Amer J Med 1986;81:791-3.

24. Martin DT, Watts PB, Newbury VS. The effect of alpine mountaineering on acute erythrocyte hemolysis. Int J Sports Med 1992:13:31-5.

25. Yoshimura $H$, Inoue $T$, Yamada $T$, Shiraki K. Anemia during hard physical training (sports anemia) and the causal mechanism with special reference to protein nutrition. WId Rev Nutr Diet 1980:35:1-86.

26. Weight I.M, Byrne M, Jacobs P. Haemolytic effects of exercisc. Clinical Science 1991:81:147-52.

27. Platt OS. Lux SE, Nathan DG. Exercise-induced hemolysis in xcrocytosis. J Clin Invest. 1981:68:631-8.

28. Robertson JD, Maughan RJ. Davidson RJL. Changes in red cell density and related indices in resonse to distance running. Eur $\mathrm{J}$ Appl Physiol 1988:57:264-9.

29. Eichner ER. Runncr's macrocytosis: A clue to footstrike hemolysis. Amer J Medicine 1985;78:321-5.

30. Refsum HE. Jordfald G. Stromme SB. Hematological changes following prolonged heavy exercise In: Jokl E, Anand RL. Stoboy H, editors. Advance in exercise physiology. Medicine and sport. Bascl (CH):Karger, 1976; 9:91-9.

31. Lorenz R, Jachmann C, Jeschke D. Bewertung von Eisenstoffwechsel-Parametern in der Sportmedizin Praktische Sport-Traumatologie und Sportmedizin 1992, 1:22-7.

32. Röcker L. Der Einfluß körperlicher Aktivität auf das Blut. 2. Auflage. In: Hollmann W, editor. Zentrale Themen der Sportmedizin. Berlin (DE): Springer Verlag, 1977; 91-111.

33. Ditzel J, Kampmann J. Whole-blood viscosity, hematocrit and plasma protein in normal subjects at different ages. Acta Physiol Scand 1971:81:264-8.

34. Itzschak Y, Silberberger A, Modan M, Adar R, Deutsch V. Hematocrit, viscosity and blood flow velocity in men and women. Israel J Med Sci 1977;13:80-2.

35. Merrill EW. Rheology of blood. Physiol Rev 1969; 49: 863-8.

36. Sander M, Gunga C, Molz B, Heyduck B, Röcker L. Zirkadiane Rhythmik des Plasmavolumens unter Berücksichtigung der volumenregulierenden Hormone (Renin, Aldosteron. Argininvasopressin und atriales natriuretisches Peptid) sowie der Plasmaproteine. Lab Med 1993;17:406-10.

37. Heilmann E, Bresch HM, Weinand H. Jung K. Anämie bei Langstreckenläufern. Dtsch Z Sportmed 1978:29:15-20.

38. Weight LM, Darge BL, Jacobs P. Athlete's pseudoanaemia. Eur J Appl Physiol 1991;62:358-62.

39. Kubanek B, Ganzoni A, Heimpel H, Nowicki L, Dörmer P, Queißer W. Einteilung der Erkrankung des erythrozytären Systems. In: Queißer.W, editor. Das Knochenmark. Stuttgait (DE): Georg Thieme Verlag, 1978, 367-71.

40. Eichner ER. The anemias of athletes. The Physician and Sportsmedicine 1986;14:122-30.

41. Chesley LC. Plasma and red all volumes during pregnancy. Am J Obstet Gynecol 1972;112:440-50.

42. Dufaux B, Hoederath A. Heck H. Schürch P. Mader A, Order U. Hollmann W. Einfluß von körperlicher Belastung auf das Serumferritin. Dtsch Z Sportmed 1980;31:253-61.

43. Jakob E, Berg A, Räther M, Keul J. Diagnostik des Eisenmangels unter besonderer Berücksichtigung des Serumferritins. Dtsch Z Sportmed 1990;41:402-10.

44. Magazanik A, Weinstein Y, Dein RA, Derin M, Schwarzmann $S$, Allalouf $D$. Iron deficiency caused by 7 weeks of intensive physical exercise. Eur J Appl Physiol 1988:57:198-202.

45. Ferguson BJ, Skinne BS, Simpson KM, Baynes RD. Cook JD. Serum transferrin receptor distinguishes the anemia of chronic disease from iron deficiency anemia. J Lab Clin Med 1992;19:38590.

46. Suominen P. Punnonen K, Rajamäki A, Irjala K. Evaluation of new immunoenzymometric assay for measuring soluble transferrin receptor to detect iron deficiency in anemic patients. Clin Chem 1997;43:1641-46.

47. Cook JD, Skikne BS, Baynes RD. Serum transferrin receptor. Annu Rev Med 1993;44:63-74. 